\title{
A US Student Reflects on Her Cuban Medical Education
}

\author{
Natalia Orihuela
}

At first I was skeptical about the opportunity for international candidates to study medicine for free in Cuba. Did the land of son cubano, cigars and sugar have the resources to host thousands of young men and women aspiring to get their medical degree? I was acquainted with the history of the country, and the prospect intrigued me. Having grown up in Mexico City and been raised by progressive parents, I had heard about Cuba's health care system and educational model. After finishing my undergraduate degree in California, I started scouting for medical colleges that advocated a holistic approach to medicine.

This search led me to Havana's Latin American School of Medicine (ELAM), an international medical school founded to train doctors devoted to practice in vulnerable, underserved communities. The Cuban government makes a global humanitarian commitment by providing full, six-year scholarships to train individuals from these same communities. After inquiring into the program, I did not hesitate. I applied and was accepted. It has become one of the most enriching experiences of my life.

When I arrived at José Martí International Airport, the scent of rain-soaked soil heralded summer in Havana. Most of the firstyear US students traveled together and completed the required 24-hour quarantine at the ELAM clinic before being transferred to the dorms. Quarantine, to monitor for spread of infectious disease, is part of the intake protocol for all foreign medical students and Cuban medical personnel returning from overseas assignments. This was our first lesson in preventive medicine.

As the days went by, students from all over the world started arriving on campus. The dormitories' close quarters gave us a chance to get to know each other better. After a six-month intensive Spanish course, the language barrier between us melted away. Living in a foreign country and studying in a language unknown to many helped us become a family and adjust to sometimes challenging conditions.

My pre-med class comprised young people from different latitudes, including Western Sahara, Palestine, Belize, Syria, Brazil, Nicaragua, Antigua, Zimbabwe, Chad and Angola. I discovered that how I perceived life back home was entirely different from the way some of my classmates did-their loved ones endured severe poverty or ruthless violence. Well-being, I realized, encompasses psychosocial conditions as well as biology.

Nonetheless, we all shared a common goal. Like most of the US students who came from historically neglected and impoverished communities, the other young people matriculating at ELAM believed that access to health care is a basic human right-no matter from where they hailed; our experiences forged friendships that affected how I understood reality and my place in the world.

The program itself, with its emphasis on community medicine, clinical skills and primary care, laid the groundwork for attaining this common goal. The pedagogical approach is team-oriented, with projects and evaluations occurring in groups. Thus, each teammate is responsible for ensuring everyone comprehends

the concepts being taught. In contrast to most US educational models, at ELAM, collective achievement is a fundamental contribution to individual academic success. Starting in first year we visited patients in their homes, inquiring into their current state of health, their sources of stress as well as their social activities.

After second year we transitioned into clinical settings at one of several Havana teaching hospitals. Extensive interaction with patients taught us to treat them within their biopsychosocial contexts. These methods helped us hone our rapport-building skills, aiding in our transition to the hospital, where patient interaction happens on a daily basis.

Moreover, students have the opportunity for in-service learning from phyisican professors throughout our six years of study. Cuban physicians strive to bequeath as much knowledge as possible to students because, in contrast to the professional competitiveness characteristic of US academic culture, they do not perceive us as future competitors, but rather as another building block in their commitment to healing. In addition to health sciences, through their examples we learned compassion, altruism and-most importantly-our responsibility as medical practitioners towards those in need, regardless of their socioeconomic status.

Now, four years into my medical studies I believe living and studying in Cuba has shaped who I am and given me a host of applicable lessons of personal and academic growth. I am convinced that no other school would have offered

me the support system, challenges and wisdom this experience entails. ELAM not only represents the place where I am getting my medical education; it is a place where I have found a family, become bilingual and made extraordinary memories.

Most importantly, however, ELAM stands as a paragon of social consciousness where science meets human compassion forging the physician I aspire to become one day. I am eager to apply everything I have learned back in the United States for I am certain I will be empowered with tools that will make a difference in my community.

Cuban medical schools take pride in educating physicians de ciencia y consciencia, (of science and conscience) because the essence of medicine lies in its humanistic nature and healing is our duty, despite any hardship. This is what I will take home. 1 -

Submitted: October 27, 2017

Approved for publication: January 10, 2018

Disclosures: None

Correspondence: norihuela@nauta.cu 